\title{
Resistencia microtraccional de capa de adhesivo contaminada con sangre
}

\section{Microtensile bond strength of adhesive layer contaminated with blood on human sound enamel}

\author{
De Nordenflycht $\mathrm{D}^{1}$, Kaplan $\mathrm{M}^{2}$, Montecinos V², Báez $\mathrm{A}^{3}$
}

\begin{abstract}
RESUMEN
Objetivo: Evaluar la influencia de la contaminación con sangre en una capa de adhesivo formada sobre esmalte humano y su posterior descontaminación con $\mathrm{NaOCl}(2.5 \%)$ y etanol $\left(70^{\circ}\right)$ en la resistencia microtraccional. Métodos: La superficie vestibular de 80 premolares humanos fue fresada para obtener superficies planas sobre las que se aplicó un adhesivo de grabado y lavado siguiendo las indicaciones del fabricante (Adper Single Bond 2, 3M ESPE). Los premolares fueron distribuidos aleatoriamente en cuatro grupos: Grupo 1 (control), Grupo 2 (contaminación con sangre), Grupo 3 (descontaminación con $\mathrm{NaOCl} 2.5 \%$ ) y Grupo 4 (descontaminación con etanol $70^{\circ}$ ). Luego, sobre cada premolar se confeccionó una corona de resina compuesta (Filtek Z350, 3M ESPE) y fueron cortados para obtener cuerpos de prueba de $1 \mathrm{~mm}^{2}$ de sección transversal, los cuales fueron termociclados $\left(5500\right.$ ciclos, $\left.5-55^{\circ} \mathrm{C}\right)$ y traccionados hasta su límite de ruptura (Micro Tensile Tester, Bisco). Los resultados fueron analizados estadísticamente (ANOVA, Scheffe, p<0.05). Resultados: La resistencia microtraccional del Grupo 1 (24.8 MPa) fue significativamente superior al resto de los grupos ( $p<0.05)$. Las diferencias entre los grupos 2,3 y 4 no fueron estadísticamente significativas ( $p>0.05)$. Conclusión: La contaminación con sangre de la capa de adhesivo interfiere significativamente en la resistencia microtraccional. La descontaminación con NaOCl o etanol no logró una recuperación de la resistencia microtraccional.
\end{abstract}

Rev. Clin. Periodoncia Implantol. Rehabil. Oral Vol. 6(3); 118-122, 2013.

Palabras clave: Sangre, contaminación, resistencia adhesiva, Adper Single Bond 2.

\begin{abstract}
Aim: To evaluate the influence of blood contamination of the adhesive layer and posterior decontamination with $\mathrm{NaOCl}(2.5 \%)$ and ethanol $\left(70^{\circ}\right)$ on microtensile bond strength on human enamel. Methods: Vestibular surfaces of eighty human premolars were grounded to obtain flat surfaces. An etch-and-rinse adhesive was applied according to the manufacturer's instructions (Adper Single bond 2, 3M ESPE). Teeth were randomly assigned into 4 groups: Group 1 (control), Group 2 (blood contamination), Group 3 (decontamination with NaOCl 2.5\%) and Group 4 (decontamination with ethanol $70^{\circ}$ ). Then, a $4 \mathrm{~mm}$ composite crown (Filtek Z350) was made and the teeth were vertically sectioned to obtain $1 \mathrm{~mm}$ cross-section stick shape specimens. Specimens were thermocycled $\left(5500\right.$ cycles, $\left.5-55^{\circ} \mathrm{C}\right)$ and pulled under tension until failure (Micro Tensile Tester, Bisco). Results were statistically analyzed (ANOVA, Scheffe's test, $p<0.05)$. Results: Microtensile bond strength in group $1(24.8 \mathrm{MPa})$ was significantly higher than groups 2,3 and $4(p<0.05)$. The differences between groups 2,3 and 4 were not significant $(p>0.05)$. Conclusion: Blood contamination significantly interferes in microtensile bond strength. Decontaminating the blood residues with $\mathrm{NaOCl}$ or ethanol did not show a recovery of bond strength.
\end{abstract}

Rev. Clin. Periodoncia Implantol. Rehabil. Oral Vol. 6(3); 118-122, 2013.

Key words: Blood, contamination, bond strength, Adper Single Bond 2.

\section{INTRODUCCIÓN}

El aislamiento absoluto de los dientes con goma dique previo a un procedimiento restaurador es fundamental para lograr un campo operatorio limpio y seco, lo cual facilita la aplicación de adhesivos y la obturación de cavidades. Sin embargo, existen algunas situaciones clínicas, como las restauraciones cervicales, donde el uso de la goma dique no es posible ${ }^{(1)}$. A pesar de la importancia del uso de la goma dique, una publicación basada en cuestionario del año 2008 muestra que un $45 \%$ de los dentistas generales en EE.UU. nunca ha utilizado la goma dique para restauraciones directas en dientes anteriores y un 39\% para restauraciones de dientes posteriores ${ }^{(2)}$. Un estudio más reciente observó una baja utilización de la goma dique durante procedimientos restauradores, sin embargo, su uso varía significativamente dependiendo del dentista, tipo de restauración y características del paciente ${ }^{(3)}$. En este contexto, es probable que ocurra una contaminación durante la aplicación del adhesivo o la inserción del material restaurador.

Clínicamente, uno de los factores que pueden afectar la retención de las restauraciones adhesivas es la contaminación del campo operatorio. Algunos de los contaminantes más comunes son saliva, sangre, astringentes y agua(4). Algunos autores han reportado una disminución significativa de la resistencia adhesiva por contaminación con sangre en dentina ${ }^{(5-8)}$ y en esmalte ${ }^{(1,9-12)}$. Esta disminución en la resistencia adhesiva podría ser atribuida al alto contenido proteico de la sangre, el cual forma una película en la superficie dental, obstruyendo la penetración del adhesivo, además de su alto contenido de agua, el cual reduce la polimerización del HEMA(1).

En la literatura se han reportado diversas estrategias para remover la sangre e, idealmente, recuperar la resistencia adhesiva, como el fresado de la superficie dental afectada, lavado con agua seguido de secado con aire, lavado con agua seguido de re-aplicación de primer, lavado con hipoclorito de sodio $(\mathrm{NaOCl})$, todos ellos con resultados heterogéneos $^{(1,8,12-16)}$. Ninguno de ellos ha evaluado la posibilidad de remover la sangre usando un agente desecante como el etanol. A pesar de la evidencia citada, el manejo de la contaminación con sangre de los procedimientos restauradores adhesivos aún no está del todo claro.

1. Cirujano Dentista. Docente del Departamento de Odontología Restauradora. Universidad Andrés Bello Viña del Mar. Chile.

2. Cirujano Dentista. Universidad Andrés Bello Viña del Mar. Chile.

3. Magíster en Odontología Restauradora. Jefe del Departamento de Odontología Restauradora. Universidad Andrés Bello Viña del Mar. Chile.

Correspondencia autor: Diego De Nordenflycht. diego.den@gmail.com. Trabajo recibido el 08/01/2013. Aprobado para su publicación el 25/05/2013. Los resultados preliminares de esta investigación fueron presentados en la Reunión anual de la IADR división Chile en Valdivia, Chile, Octubre de 2010. 
El objetivo de la presente investigación es evaluar el efecto de la contaminación con sangre en una capa de adhesivo formada sobre esmalte humano y el efecto de la descontaminación de esta capa utilizando $\mathrm{NaOCl}(2.5 \%)$ y etanol $\left(70^{\circ}\right)$ en la resistencia microtraccional. La hipótesis nula testeada fue que los procedimientos de descontaminación no afectan la resistencia adhesiva.

\section{MATERIALES Y MÉTODO}

Se utilizaron 80 premolares definitivos, humanos, sanos, recientemente extraídos por motivos ortodóncicos, obtenidos de veinte pacientes (20 a 28 años de edad) de ambos géneros (12 hombres y 8 mujeres) que voluntariamente, y sin pago de por medio, aceptaron donar sus dientes, manifestado verbalmente y de forma escrita en un consentimiento informado. Los dientes fueron almacenados en suero fisiológico $0.9 \%$ por una semana desde la exodoncia hasta la etapa experimental. No se utilizaron desinfectantes en su almacenamiento para evitar potenciales efectos perjudiciales en el procedimiento adhesivo. A todos los dientes se les removió el material orgánico mediante instrumentación periodontal manual (J30-33 JK5, American Eagle, Missoula, EE.UU.) y fueron limpiados con escobilla de profilaxis a baja velocidad con agua potable del grifo. Posteriormente, las raíces de los dientes fueron incluidas en cubos de acrílico de autocurado (Acrílico Rosado, Marche, Santiago, Chile) para facilitar la manipulación y posterior montaje de los dientes. Las superficies vestibulares de los dientes fueron desgastadas mediante fresado a alta velocidad bajo refrigeración con agua (G842-314-113-524-014-ML, Coltene Whaledent, Altstatten, Suiza) y posteriormente pulidas manualmente con lija al agua grano 600 para lograr una superficie de esmalte plana y regular. Sobre estas superficies preparadas se aplicó y fotopolimerizó con luz halógena (Coltolux LED, Coltene Whaledent, Cuyahoga Falls, $\mathrm{OH}, \mathrm{EE}$. UU.) una capa de un adhesivo de grabado y lavado siguiendo las indicaciones del fabricante (Adper Single Bond 2, 3M ESPE, St Paul, MN, EE.UU.). Posteriormente, los dientes fueron distribuidos aleatoriamente en cuatro grupos $(n=20)$, estableciéndose los siguientes grupos (ver Figura 1):

\section{- Grupo 1 (Control): Sin contaminación de la capa de adhesivo.}

- Grupo 2 (Contaminación con sangre): La capa de adhesivo fue contaminada con sangre humana venosa fresca. La sangre fue recolectada de una mujer de 22 años de edad, voluntaria, utilizando una jeringa desechable de $10 \mathrm{~mL}$. Se aplicó y distribuyó una gota de sangre sobre la capa de adhesivo con aguja desechable. El exceso de sangre fue removido utilizando una esfera de $4 \mathrm{~mm}$ de diámetro de algodón estéril. La sangre residual fue secada con un suave chorro de aire utilizando la jeringa triple.

- Grupo 3 (Descontaminación con $\mathrm{NaOCl} 2.5 \%$ ): La capa de adhesivo fue contaminada con sangre humana siguiendo el mismo procedimiento descrito para el Grupo 2. Luego de secada la sangre residual, una esfera de algodón de $4 \mathrm{~mm}$ de diámetro embebida en hipoclorito de Sodio $2.5 \%$ $(\mathrm{NaOCl})$ fue ligeramente frotada por 10 s sobre la capa de adhesivo contaminada. EI $\mathrm{NaOCl}$ residual fue secado con un suave chorro de aire utilizando la jeringa triple.

- Grupo 4 (Descontaminación con etanol $70^{\circ}$ ): La capa de adhesivo fue contaminada con sangre humana siguiendo el mismo procedimiento descrito para el Grupo 2. Luego de secada la sangre residual, una esfera de algodón de $4 \mathrm{~mm}$ de diámetro embebida en etanol $70^{\circ}$ fue ligeramente frotada por 10 s sobre la capa de adhesivo contaminada. El etanol residual fue secado con un suave chorro de aire utilizando la jeringa triple.

Sobre todas superficies tratadas se confeccionó una corona cilíndrica de resina compuesta de 4mm (Filtek Z350, color A1, 3M ESPE, St Paul MN, EE.UU.). La resina compuesta fue aplicada en incrementos de $1 \mathrm{~mm}$ y cada incremento fue fotopolimerizado con luz halógena por 20s (Coltolux LED, Coltene Whaledent, Cuyahoga Falls, OH, EE.UU.). Luego, los dientes fueron seccionados para obtener cuerpos de prueba rectangulares, de $1 \mathrm{~mm}^{2}$ de sección transversal, utilizando una sierra de precisión (Isomet 1000, Buehler, Lake Bluff, IL, EE.UU.) y medidos con calibrador digital. Una variación de $0.1 \mathrm{~mm}$ se consideró aceptable. Los cuerpos de prueba fueron analizados con magnificación $(2 \mathrm{x})$ para descartar aquellos que tuvieran defectos en la capa de adhesivo. Al menos 4 cuerpos de prueba por cada diente fueron seleccionados. Los cuerpos de prueba seleccionados fueron termociclados por 5500 ciclos entre $5^{\circ} \mathrm{C}$ y $55^{\circ} \mathrm{C}$ (Mastercycler personal, Eppendorf, Hamburgo, Alemania) siguiendo una modificación de la especificación ISO $11405^{(17)}$ y luego fueron traccionados hasta su límite de ruptura (Micro Tensile Tester, Bisco Inc, Schaumburg, IL, EE.UU.) a una velocidad de $1 \mathrm{~mm} /$ min. La resistencia microtraccional fue expresada en MPa y calculada como la fuerza de tracción dividida por el área adherida. Los valores obtenidos para los cuerpos de prueba de un mismo diente fueron promediados y se consideró un valor por cada diente para el análisis de los resultados.

Luego de fracturados, los cuerpos de prueba fueron observados con microscopio estereoscopico a un aumento de $4 x$ (SZ51, Olympus, Hamburgo, Alemania) para determinar el modo de falla post prueba: adhesiva (falla entre el esmalte y el adhesivo), cohesiva (falla dentro del esmalte o la resina compuesta) y mixta (falla adhesiva y cohesiva en el mismo cuerpo de prueba). Los resultados fueron analizados con test ANOVA para evaluar la influencia de la contaminación con sangre y los procedimientos descontaminantes en la resistencia microtraccional, y se realizaron comparaciones múltiples con test post hoc de Scheffe con el software SPSS 17 (SPSS Inc., Chicago IL, EE.UU.). El nivel de significancia fue establecido como $p=0.05$. Para analizar si la dispersión de valores de resistencia microtraccional era afectada por la contaminación con sangre, se sometió al test de la $\mathrm{F}$ las desviaciones estándar de los grupos, comparando cada grupo contra el control.

\section{RESULTADOS}

Las medias y desviaciones estándar de los valores de resistencia microtraccional encontrados se muestran en la Tabla 1. La media de resistencia microtraccional mayor se encontró en el grupo control (24.8 MPa). El resultado del análisis de varianza (ANOVA) muestra que las diferencias encontradas entre los grupos son significativas $[F(3,73)=8.482, p=0.00007]$. El test de Scheffe muestra que los valores de resistencia microtraccional obtenidos en el Grupo control fueron significativamente mayores a los valores de los Grupos 2, 3 y 4 (ver Tabla 1). Las comparaciones múltiples entre los Grupos 2, 3 y 4 muestra que las diferencias encontradas no fueron significativas $(p>0.05)$, por lo tanto, se acepta la hipótesis nula. El valor mínimo registrado fue de $0.4 \mathrm{MPa}$, encontrado en los Grupos 2, 3 y 4. $\mathrm{Ni}$ el etanol ni el hipoclorito de sodio pudieron recuperar la resistencia microtraccional luego de la contaminación con sangre (ver Figura 2). El análisis de la dispersión de los datos mostró diferencias significativas de las desviaciones estándar con respecto al Grupo control para Grupo 2 (F-test: [F=2.859, $p=0.027]$ ), Grupo 3 (F-test: [F=3.047, $p=0.019]$ ) mientras que esta diferencia no fue significativa para el Grupo 4 (F-test: $[F=1.909, p=0.168])$.

La incidencia de los modos de falla se muestra en la Figura 3. El modo de falla más frecuente fue la falla adhesiva $(64.1 \%)$ seguido de la falla mixta $(21.3 \%)$ y la falla cohesiva (14.6\%). En los cuerpos de prueba de los Grupos 2, 3 y 4 se observó una delgada línea marrón entre el adhesivo y la resina compuesta que indica la presencia de residuos de sangre. 


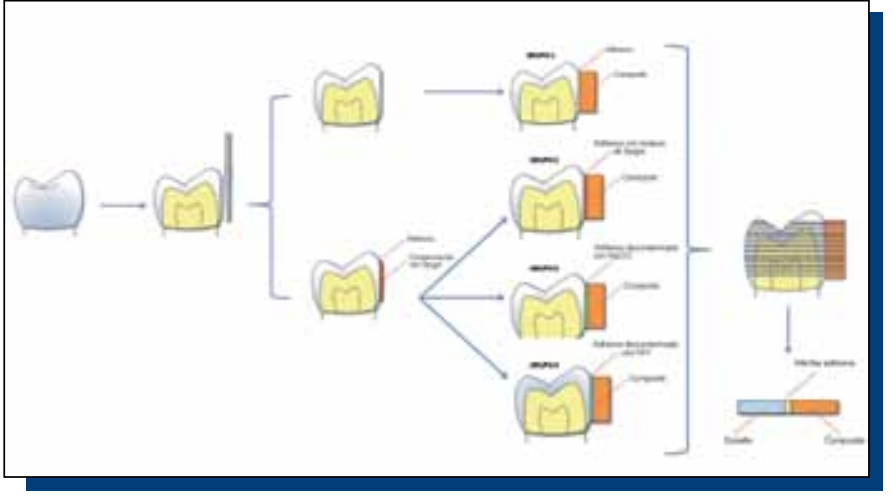

Figura 1. llustración esquemática del procesado de los cuerpos de prueba para resistencia microtraccional.

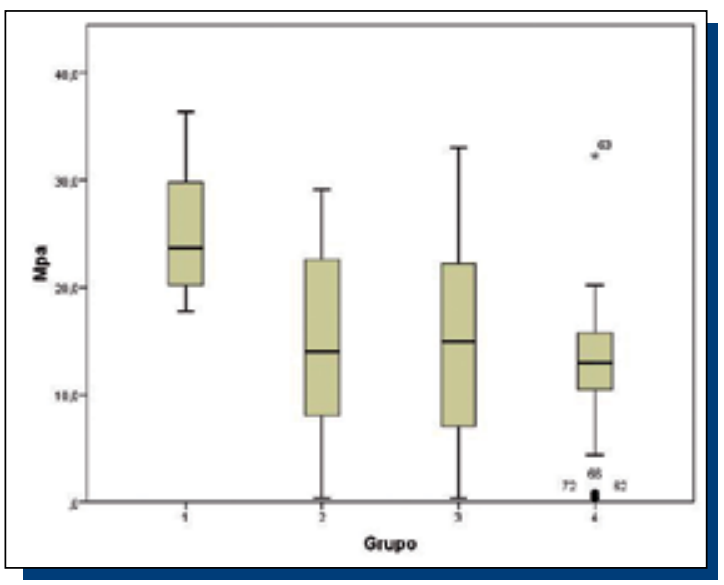

Figura 2. Distribución de los valores de resistencia microtraccional por grupo.

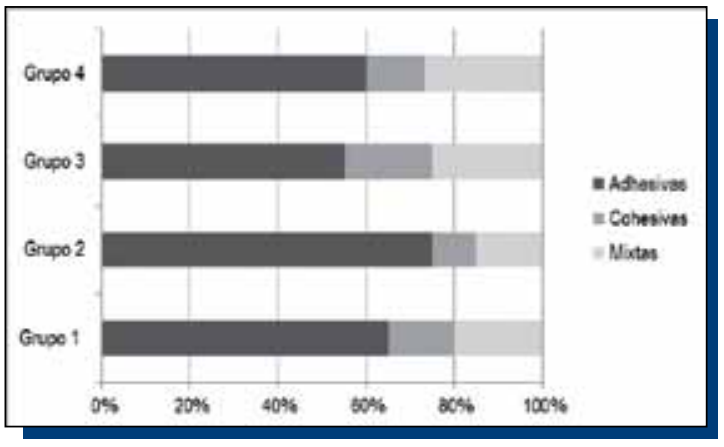

Figura 3. Incidencia de los modos de falla post prueba de resistencia microtraccional.

Tabla 1. Estadísticos descriptivos, comparaciones de las medias y desviación estándar, y resultado del test de Scheffe para cada grupo versus grupo control.

\begin{tabular}{|l|c|c|c|}
\hline \hline & Media & $\begin{array}{c}\text { Desviación } \\
\text { estándar }\end{array}$ & $\begin{array}{c}\text { p valor } \\
\text { versus control }\end{array}$ \\
\hline $\begin{array}{l}\text { Grupo 1 } \\
\text { (Control) }\end{array}$ & 24.8 & 5.5 & - \\
\hline $\begin{array}{l}\text { Grupo 2 } \\
\text { (Contaminación con sangre) }\end{array}$ & 14.6 & 9.3 & 0.003 \\
\hline $\begin{array}{l}\text { Grupo 3 } \\
\text { (Descontaminación con } \\
\text { NaOCl 2.5\%) }\end{array}$ & 15.3 & 9.6 & 0.006 \\
\hline $\begin{array}{l}\text { Grupo 4 (Descontaminación } \\
\text { con etanol 70 })\end{array}$ & 12.5 & 7.9 & 0.000 \\
\hline
\end{tabular}

Tabla 2. Resultados principales de las publicaciones sobre la influencia de la contaminación con sangre en la adhesión a esmalte (periodo 2001-2011).

\begin{tabular}{|c|c|c|c|c|}
\hline Autor & Año & $\begin{array}{l}\text { Momento de la } \\
\text { contaminación }\end{array}$ & $\begin{array}{c}\text { Agente } \\
\text { adhesivo }\end{array}$ & $\begin{array}{l}\text { Reducción de } \\
\text { la resistencia } \\
\text { adhesiva (\%) }\end{array}$ \\
\hline \multirow{3}{*}{\begin{tabular}{|l|l|}
$\begin{array}{l}\text { Oonsombat } \\
\text { cols }^{(9)}\end{array}$ & $y$
\end{tabular}} & \multirow[t]{3}{*}{2003} & Antes de imprimar & \multirow{3}{*}{$\begin{array}{l}\text { Angel I (3M } \\
\text { ESPE) }\end{array}$} & 55.0 \\
\hline & & $\begin{array}{l}\text { Después de } \\
\text { imprimar }\end{array}$ & & 81.7 \\
\hline & & Antes y después & & 91.7 \\
\hline \multirow[t]{3}{*}{$\begin{array}{l}\text { Oztoprak } \\
\text { cols }^{(10)}\end{array}$} & \multirow[t]{3}{*}{2007} & $\begin{array}{l}\text { Después de } \\
\text { grabar }\end{array}$ & $\begin{array}{c}\text { Transbond } \\
\text { XT (3M } \\
\text { Unitek) }\end{array}$ & 79.8 \\
\hline & & $\begin{array}{l}\text { Después de } \\
\text { imprimar }\end{array}$ & $\begin{array}{l}\text { Transbond } \\
\text { Plus SEP } \\
\text { (3M Unitek) }\end{array}$ & 61.6 \\
\hline & & $\begin{array}{l}\text { Después de } \\
\text { grabar }\end{array}$ & $\begin{array}{l}\text { Assure } \\
\text { (Reliance) }\end{array}$ & 58.4 \\
\hline \multirow[t]{2}{*}{$\begin{array}{l}\text { Sayinsu } \\
\text { cols }^{(11)}\end{array}$} & \multirow[t]{2}{*}{2007} & $\begin{array}{l}\text { Antes del adhe- } \\
\text { sivo }\end{array}$ & \multirow[t]{2}{*}{$\begin{array}{l}\text { Biscover } \\
\text { (Bisco) }\end{array}$} & 62.8 \\
\hline & & $\begin{array}{l}\text { Después del } \\
\text { adhesivo }\end{array}$ & & 7.2 \\
\hline \multirow[t]{2}{*}{$\begin{array}{l}\begin{array}{l}\text { Faltermeier } \\
\text { cols }^{(13)}\end{array} \\
y\end{array}$} & \multirow[t]{2}{*}{2007} & \multirow[t]{2}{*}{$\begin{array}{l}\text { Después de } \\
\text { imprimar }\end{array}$} & $\begin{array}{c}\text { Transbond } \\
\text { XT (3M } \\
\text { Unitek) }\end{array}$ & 72.8 \\
\hline & & & $\begin{array}{c}\text { Transbond } \\
\text { MIP (3M } \\
\text { Unitek) }\end{array}$ & 23.8 \\
\hline \multirow[t]{2}{*}{ Santos y cols ${ }^{(21)}$} & \multirow[t]{2}{*}{2010} & \multirow[t]{2}{*}{$\begin{array}{l}\text { Después de } \\
\text { imprimar }\end{array}$} & $\begin{array}{l}\text { Transbond } \\
\text { MR Plus } \\
\text { (3M Unitek) }\end{array}$ & 22.2 \\
\hline & & & $\begin{array}{c}\text { Transbond } \\
\text { XT (3M } \\
\text { Unitek) }\end{array}$ & 94.4 \\
\hline \multirow[t]{4}{*}{ 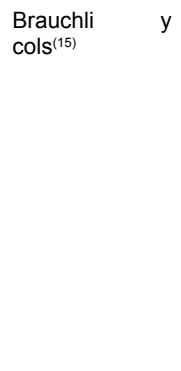 } & \multirow[t]{4}{*}{2010} & \multirow[t]{4}{*}{$\begin{array}{l}\text { Después del } \\
\text { adhesivo }\end{array}$} & $\begin{array}{c}\text { Transbond } \\
\text { XT (3M } \\
\text { Unitek) }\end{array}$ & 80.5 \\
\hline & & & $\begin{array}{c}\text { Transbond } \\
\text { MIP (3M } \\
\text { Unitek) }\end{array}$ & 96.4 \\
\hline & & & $\begin{array}{l}\text { Transbond } \\
\text { Plus SEP } \\
\text { (3M Unitek) }\end{array}$ & 87.6 \\
\hline & & & $\begin{array}{l}\text { Fuji Ortho } \\
\text { LC (GC) }\end{array}$ & 82.2 \\
\hline \multirow[t]{2}{*}{$\begin{array}{l}\text { de Carvahlo } \\
\text { Mendoça }{ }^{(1)}\end{array}$} & \multirow[t]{2}{*}{2010} & $\begin{array}{l}\text { Antes del } \\
\text { adhesivo }\end{array}$ & $\begin{array}{l}\text { Clearfil } \\
\text { SE Bond } \\
\text { (Kuraray) }\end{array}$ & 22.9 \\
\hline & & $\begin{array}{l}\text { Después del } \\
\text { adhesivo }\end{array}$ & & 100 \\
\hline \multirow[t]{2}{*}{$\begin{array}{l}\text { Khosravanifard } \\
\text { y cols }{ }^{(22)}\end{array}$} & \multirow[t]{2}{*}{2010} & $\begin{array}{l}\text { Después de } \\
\text { imprimar }\end{array}$ & $\begin{array}{c}\text { iBonDGI } \\
\text { (Heraus } \\
\text { Kulzer) }\end{array}$ & 29.2 \\
\hline & & $\begin{array}{l}\text { Después de } \\
\text { grabar }\end{array}$ & $\begin{array}{l}\text { Fuji Ortho } \\
\text { LC (GC) }\end{array}$ & 89.3 \\
\hline \multirow[t]{3}{*}{\begin{tabular}{|l|l|}
$\begin{array}{l}\text { Sfondrini } \\
\text { cols }^{(23)}\end{array}$ & $y$
\end{tabular}} & \multirow[t]{3}{*}{2011} & Antes de imprimar & \multirow[t]{3}{*}{$\begin{array}{c}\text { Transbond } \\
\text { XT (3M } \\
\text { Unitek) }\end{array}$} & 89.8 \\
\hline & & $\begin{array}{l}\text { Después de } \\
\text { imprimar }\end{array}$ & & 82.0 \\
\hline & & Antes y después & & 86.2 \\
\hline $\begin{array}{l}\text { Tachibana } \\
\text { cols }^{(12)}\end{array}$ & 2011 & Antes de imprimar & $\begin{array}{l}\text { Clearfil } \\
\text { S3 Bond } \\
\text { (Kuraray) }\end{array}$ & 91.3 \\
\hline $\begin{array}{l}\text { De Nordenflycht } \\
\text { y cols }\end{array}$ & 2013 & $\begin{array}{l}\text { Después del } \\
\text { adhesivo }\end{array}$ & $\begin{array}{l}\text { Single Bond } 2 \\
\text { (3M ESPE) }\end{array}$ & 41.1 \\
\hline
\end{tabular}


Tabla 3. Procedimientos de descontaminación de la sangre, descritos en la literatura, con el fin de recuperar la resistencia adhesiva posterior a la contaminación con sangre.

\begin{tabular}{|c|c|c|c|c|}
\hline Autor & Año & $\begin{array}{c}\text { Restauración o } \\
\text { bracket }\end{array}$ & Sustrato & $\begin{array}{l}\text { Procedimiento de } \\
\text { descontaminación } \\
\text { efectivo }\end{array}$ \\
\hline $\begin{array}{l}\text { Eiriksson y } \\
\text { cols }^{(19)}\end{array}$ & 2004 & Restauración & Restauración & $\begin{array}{l}\text { Espray de agua/aire } \\
\text { y re-aplicación de } \\
\text { adhesivo }\end{array}$ \\
\hline Yoo y Pereira ${ }^{(8)}$ & 2006 & Restauración & Dentina & $\begin{array}{l}\text { No fue posible } \\
\text { sobrellevar el efecto } \\
\text { de la sangre }\end{array}$ \\
\hline $\begin{array}{l}\text { Raffaini y } \\
\text { cols }^{(14)}\end{array}$ & 2008 & Restauración & Dentina & Enjuague con agua \\
\hline Chang y $\operatorname{cols}^{(16)}$ & 2010 & Restauración & Dentina & $\begin{array}{l}\text { No fue posible } \\
\text { sobrellevar el efecto } \\
\text { de la sangre }\end{array}$ \\
\hline $\begin{array}{c}\text { Khosravanifard } \\
y_{\text { cols }}^{(22)}\end{array}$ & 2010 & Bracket & Esmalte & $\begin{array}{l}\text { Enjuague, secado y } \\
\text { re-grabado ácido }\end{array}$ \\
\hline $\begin{array}{l}\text { Brauchli y } \\
\text { cols }^{(15)}\end{array}$ & 2010 & Restauración & Esmalte & $\begin{array}{l}\text { Enjuague, secado y re } \\
\text { aplicación de primer. }\end{array}$ \\
\hline $\begin{array}{c}\text { Tachibana y } \\
\text { cols }^{(12)}\end{array}$ & 2011 & Restauración & Dentina/esmalte & Chorro de agua \\
\hline
\end{tabular}

\section{DISCUSIÓN}

La adhesión al esmalte es unos de los procedimientos restauradores más predecibles de la odontología restauradora adhesiva moderna. La escaza cantidad de agua y la morfología de los prismas del esmalte han permitido una íntima adaptación entre la resina hidrófoba del adhesivo y el esmalte acondicionado desde que Buonocore (1955) estableció el procedimiento del grabado ácido del esmalte ${ }^{(18)}$. Pero incluso este sólido mecanismo de unión requiere de un campo operatorio limpio y seco debido a que algunos agentes contaminantes, como la sangre, pueden tener un efecto perjudicial en la unión adhesivoesmalte, disminuyendo la resistencia adhesiva ${ }^{(1,9-12)}$. Cuando ocurre una contaminación, la menor resistencia adhesiva lograda llevará a infiltración marginal, formación de caries secundaria y tinción de los márgenes de la restauración ${ }^{(19)}$. La mayoría de las veces, esta contaminación puede evitarse mediante el uso de goma dique, sin embargo, recientes publicaciones indican que la mayoría de los clínicos no suele utilizarla(2).

Varios autores han evaluado el efecto perjudicial de la contaminación con sangre durante los procedimientos restauradores adhesivos ${ }^{(1,13,15,20)}$. Los resultados principales de los artículos más relevantes publicados en Medline y Scopus (términos de búsqueda [bond strength AND blood] y [bond strength AND blood contamination], en revistas dentales, periodo 2001-2011) sobre la influencia de la contaminación con sangre en la resistencia adhesiva se muestran en la Tabla 2, los cuales han sido simplificados para facilitar las comparaciones entre los resultados del presente estudio y los publicados en la literatura. A pesar de esto, las comparaciones aún siguen siendo difíciles debido a que los artículos contienen muchas diferencias en términos de diseño experimental, objeto de estudio (restauración o bracket ortodóncico), sistema adhesivo, tipo de sangre (fresca o heparinizada), entre otras. Respecto a esto último, se ha demostrado que el uso de sangre fresca es más adecuado que la sangre heparinizada para investigaciones de laboratorio que buscan estudiar la contaminación con sangre en la adhesión a tejidos dentales ${ }^{(6)}$, por lo que en la presente investigación no se utilizaron anticoagulantes con el fin de evitar efectos no deseados. Los resultados de la presente investigación muestran que la reducción de la resistencia adhesiva observada en el grupo de contaminación con sangre $(41.1 \%)$ es consistente con los resultados encontrados en la literatura (22.2-100\%). Un hallazgo interesante es que el momento de la contaminación dentro de los pasos de la aplicación del adhesivo es determinante para la efectividad del sellado del adhesivo. Damé y cols. ${ }^{(20)}$ observó que la contaminación con sangre seguido de la fotopolimerización del adhesivo es más perjudicial que la contaminación previa a la fotopolimerización.

En varias situaciones clínicas, la contaminación con sangre ocurre después de la formación y fotopolimerización de la capa de adhesivo. Con el objetivo de evitar los efectos negativos de la contaminación con sangre en las restauraciones adhesivas, el clínico debe decidir entre dos alternativas: Realizar nuevamente los pasos para establecer una nueva capa de adhesivo o usar algún procedimiento de descontaminación ${ }^{(20)}$. En la literatura es posible encontrar varias alternativas para contrarrestar el efecto de la sangre en la adhesión a tejidos dentales. Algunos de ellos son: secado, lavado con agua y secado(1), frotado con papel absorbente, grabado con ácido, aplicación de $\mathrm{NaOCl} 10 \%\left({ }^{(20)}\right.$, re-aplicación de primers autograbantes ${ }^{(16)}$, pero no existe consenso respecto a cuál es el mejor procedimiento de descontaminación. La Tabla 3 muestra los resultados principales de las investigaciones in vitro más relevantes publicadas en los últimos 10 años indexadas en Medline y Scopus.

Nuestros resultados muestran que la contaminación con sangre de la capa de adhesivo Adper Single Bond 2 formada en esmalte disminuye significativamente la resistencia microtraccional, y la descontaminación de esta capa no logró recuperar los valores de resistencia adhesiva. La descontaminación con $\mathrm{NaOCl} 2.5 \%$ recuperó parte de la resistencia adhesiva pero no logró ser significativo. Más aún, la descontaminación con etanol $70^{\circ}$ provocó una pequeña disminución en la resistencia microtraccional comparado con el grupo de contaminación con sangre, probablemente asociado a la solubilización de los monómeros remanentes en la capa inhibida por oxígeno presente en la superficie del adhesivo, que permite la unión a otros monómeros de materiales resinosos restauradores. Otro aspecto importante, que podría tener consecuencias clínicas, es el aumento de la desviación estándar de los valores de resistencia adhesiva observado para los grupos con contaminación con sangre. Esto podría indicar que el resultado clínico se vuelve más impredecible por la alta variabilidad de los resultados. Una posible explicación es que la contaminación del adhesivo reduce la reactividad de los monómeros superficiales no-polimerizados que son necesarios para establecer una unión predecible con la resina compuesta. Dame y cols. ${ }^{(20)}$ sugieren la aplicación de una nueva capa de adhesivo para recuperar esta actividad de los monómeros superficiales. Estudios previos sugieren lavar con agua y luego re-aplicación de adhesivo frente a una contaminación con sangre ${ }^{(19)}$.

La influencia de la contaminación con sangre en la adhesión a tejidos dentales puede deberse a dos características fundamentales de la sangre: Es un fluido rico en proteínas y rico en agua. Se ha sugerido que la presencia proteínas plasmáticas, en conjunto con macromoléculas como el fibrinógeno y las plaquetas, pueden formar una delgada película superficial que altera la adhesión ${ }^{(12)}$. Algunos autores han propuesto que esta presencia de proteínas podría influir en la resistencia adhesiva ${ }^{(4)}$. Por esto es que se decidió descontaminar la capa de adhesivo contaminada con un agente desproteinizante $(\mathrm{NaOCl})$ y con un agente deshidratante (etanol).

La mayoría de las publicaciones revisadas que han evaluado el efecto de la sangre en la adhesión, lo han hecho en relación a la unión de brackets ortodóncicos (ver Tabla 2). Sin embargo, la contaminación de la capa de adhesivo durante los procedimientos restauradores es altamente relevante. La escaza cantidad de publicaciones sobre la contaminación con sangre en procedimientos restauradores, y la falta de consenso sobre su manejo clínico, indican que este problema no ha sido completamente estudiado aún.

\section{CONCLUSIÓN}

Considerando las limitaciones propias de un estudio in vitro, podemos concluir que la contaminación con sangre de una capa de adhesivo polimerizada disminuye significativamente su resistencia microtraccional. La descontaminación de esta capa con hipoclorito de sodio $2.5 \%$ o etanol $70^{\circ}$ no recuperaron las resistencia microtraccional. Desde un punto de vista clínico, sugerimos que en caso de contaminación con sangre de la capa de adhesivo, esta debe ser removida, controlar el sangrado y formar una nueva capa de adhesivo. 


\section{AGRADECIMIENTOS}

Los autores de la presente investigación agradecen al Dr. Claudio Gandarillas Fuentes por su desinteresada y valiosa contribución en el diseño y confección de las figuras utilizadas para esta investigación.

\section{CONFLICTO DE INTERESES}

Los autores declaran no tener ninguna vinculación comercial ni financiera con cualquiera de los productos utilizados para la realización de esta investigación. La presente investigación fue realizada mediante autofinanciamiento.

\section{REFERENCIAS BIBLIOGRÁFICAS}

1. De Carvalho Mendonça EC, Vieira SN, Kawaguchi FA, Powers J, Matos AB. Influence of blood contamination on bond strength of a self-etching system. Eur $J$ Dent, 2010; 4: 280-286.

2. Hill EE, Rubel BS. Do dental educators need to improve their approach to teaching rubber dam use? J Dent Educ, 2008; 72: 1177-1181.

3. Gilbert GH, Litaker MS, Pihlstrom DJ, Amundson CW, Gordan VV. DPBRN Collaborative Group. Rubber dam use during routine operative dentistry procedures: Findings from the Dental PBRN. Oper Dent, 2010; 35: 491-499.

4. Pinzon LM, Powers JM, O'Keefe KL, Dusevish V, Spencer P, Marshall GW. Effect of mucoprotein on the bond strength of resin composite to human dentin. Odontology, 2011; 99: 119-128.

5. Abdalla Al, Davidson CL. Bonding efficiency and interfacial morphology of onebottle adhesives to contaminated dentin surfaces. Am J Dent, 1998; 11: 281-285.

6. Dietrich T, Kraemer ML, Roulet JF. Blood contamination and dentin bondingeffect of anticoagulant in laboratory studies. Dent Mater, 2002; 18: 159-162.

7. Van Schalkwyk JH, Botha FS, van der Vyver PJ, de Wet FA, Botha SJ. Effect of biological contamination on dentine bond strength of adhesive resins. SADJ, 2003; 58: 143-147.

8. Yoo HM, Pereira PN. Effect of blood contamination with 1-step self-etching adhesives on microtensile bond strength to dentin. Oper Dent, 2006; 31: 660-665.

9. Oonsombat C, Bishara SE, Ajlouni R. The effect of blood contamination on the shear bond strength of orthodontic brackets with the use of a new self-etch primer. Am J Orthod Dentofacial Orthop, 2003; 123: 547-550.

10. Oztoprak MO, Isik F, Sayinsu K, Arun T, Aydemir B. Effect of blood and saliva contamination on shear bond strength of brackets bonded with 4 adhesives. Am J Orthod Dentofacial Orthop, 2007; 131: 238-242.

11. Sayinsu K, Isik F, Sezen S, Aydemir B. Effect of blood and saliva contamination on bond strength of brackets bonded with a protective liquid polish and a light-cured adhesive. Am J Orthod Dentofacial Orthop, 2007; 131: 391-394.

12. Tachibana A, Castanho GM, Vieira SN, Matos AB. Influence of blood contamination on bond strength of a self-etching adhesive to dental tissues. $J$ Adhes Dent, 2011; 13: 349-358.
13. Faltermeier A, Behr M, Rosentritt $M$, Reicheneder $C$, Müssig D. An in vitro comparative assessment of different enamel contaminants during bracket bonding. Eur J Orthod, 2007; 29: 559-563.

14. Raffaini MS, Gomes-Silva JM, Torres-Mantovani CP, Palma-Dibb RG, Borsatto MC. Effect of blood contamination on the shear bond strength at resin/dentin interface in primary teeth. Am J Dent, 2008; 21: 159-162.

15. Brauchli L, Eichenberger $M$, Steineck $M$, Wichelhaus $A$. Influence of decontamination procedures on shear forces after contamination with blood or saliva. Am J Orthod Dentofacial Orthop, 2010; 138: 435-441.

16. Chang SW, Cho BH, Lim RY, Kyung SH, Park DS, Oh TS, Yoo HM. Effects of blood contamination on microtensile bond strength to dentin of three self-etch adhesives. Oper Dent, 2010; 35: 330-336.

17. ISO. Dental materials - Testing of adhesion to tooth structure. Technical Specification, 2003; № 11405.

18. Buonocore MG. A simple method of increasing the adhesión of acrylic filling materials to enamel surfaces. J Dent Res, 1955; 34: 849-853.

19. Eiriksson SO, Pereira PN, Swift EJ, Heymann HO, Sigurdsson A. Effects of blood contamination on resin-resin bond strength. Dent Mater, 2004; 20: 184-190.

20. Damé JLD, Torriai DD, Demarco FF, Goettems ML, Rodrigues-Junior SA, Piva E. Effect of blood contamination and decontamination procedures on marginal adaptation and bond strength of composite restorations. Rev Odonto Ciênc, 2009; 24: $283-289$

21. Santos BM, Pithon MM, Ruellas AC, Sant'Anna EF. Shear bond strength of brackets bonded with hydrophilic and hydrophobic bond systems under contamination. Angle Orthod, 2010; 80: 963-967.

22. Khosravanifard B, Rakhshan V, Saadatmand A. Effects of blood and saliva contamination on shear bond strength of metal orthodontic brackets and evaluating certain methods for reversing the effect of contamination. Orthodontic Waves, 2010; 69: 156-163

23. Sfondrini MF, Gatti S, Scribante A. Effect of blood contamination on shear bond strength of orthodontic brackets and disinclusion buttons. Br J Oral Maxillofac Surg, 2011; 49: 404-408. 